\title{
Performance Analysis of Hybrid 5G-GNSS Localization
}

\author{
(This is a preprint of an accepted article scheduled to be presented at \\ The Asilomar Conference on Signals, Systems, and Computers, to be held October 28-31, 2018, \\ in Asilomar Grounds in Pacific Grove, CA, USA.)

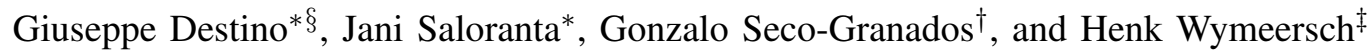 \\ *University of Oulu, Oulu, Finland \\ ${ }^{\dagger}$ Universitat Autonoma de Barcelona, Barcelona, Spain \\ $\ddagger$ Chalmers University of Technology, Gothenburg, Sweden \\ $\S$ King's College London, London, United Kingdom \\ email: \{giuseppe.destino, jani.saloranta\}@oulu.fi, henkw@chalmers.se, gonzalo.seco@uab.cat
}

\begin{abstract}
We consider a novel positioning solution combining millimeter wave $(\mathrm{mmW})$ 5G and Global Navigation Satellite System (GNSS) technologies. The study is carried out theoretically by deriving the Fisher Information Matrix (FIM) of a combined 5G-GNSS positioning system and, subsequently, the position, rotation and clock-bias error lower bounds. We pursue a two-step approach, namely, computing first the FIM for the channel parameters, and then transforming it into the FIM of the position, rotation and clock-bias. The analysis shows advantages of the hybrid positioning in terms of $i$ ) localization accuracy, ii) coverage, $i i i$ ) precise rotation estimation and $i v$ ) clock-error estimation. In other words, we demonstrate that a tight coupling of the two technologies can provide mutual benefits.
\end{abstract}

\section{INTRODUCTION}

It is well-known that accurate radio-based positioning is the enabler of many location-based services related for instance to safety, intelligent transportation, entertainment, industry automation, robotics and remote operation services [1], [2]. However, Global Navigation Satellite System (GNSS), which is a term that comprises GPS and Galileo among other systems, constitutes still today the only wide-spread reliable solution thanks to its high precision and coverage [3]. In [4], it is shown that also millimeter wave $(\mathrm{mmW}) 5 \mathrm{G}$ technology can be used for accurate positioning, especially in indoor environment where GNSS solutions are not feasible. For this reason positioning has gained interest in 3GPP, searching for a standardized way to improve LTE-techniques using the socalled New Radio (NR) and, potentially, GNSS jointly.

In this paper we study a novel solution combining $\mathrm{mmW} 5 \mathrm{G}$ and GNSS technologies, extending the work in [5], with the objective of improving GNSS coverage and, overall, enabling better localization performance. The study is carried on from a theoretical perspective deriving the Fisher Information Matrix (FIM) [6] of a hybrid 5G-GNSS localization estimation and, subsequently, the position and rotation error lower bound. We pursue a two-step approach, namely, computing first the FIM for the position-related channel parameters and after, the Equivalent Fisher Information Matrix (EFIM) for position and rotation. Also, we look at the UE's clock-bias estimation, which is the enabler for Time-of-Arrival (ToA) estimation.
The reminder of this paper is organized as follows. In Section II we define the 5G-GNSS system model. In Sections

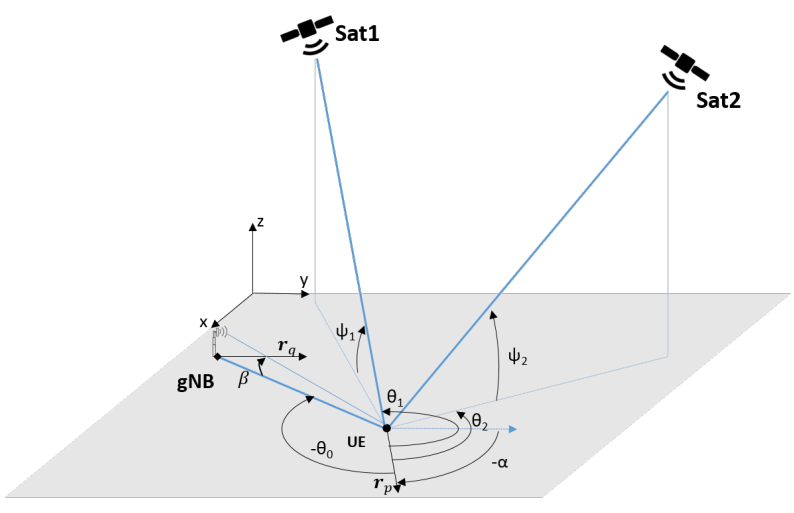

Fig. 1. Transmission links and geometry of the scenario including a $5 \mathrm{G}$ station (gNB) at location $\mathbf{q}$ and receiver (UE) at location $\mathbf{p}$ as well as two satellite stations at location $\mathbf{q}_{1}$ and $\mathbf{q}_{2}$, respectively. All links are assumed in LOS. The angle $\alpha$ is the rotation of the UE with respect to the gNB heading.

III and IV, we tackle the 5G-GNSS position-rotation bound by providing the key derivation steps along with numerical results. Finally, in Section $\mathrm{V}$ useful insights are drawn.

\section{SySTEM MODEL}

We consider an hybrid positioning system including GNSS and $\mathrm{mmW} 5 \mathrm{G}$ technology as depicted in Figure 1. More specifically, we assume a UE connected to both a $5 \mathrm{G}$ station (gNB) and $S$ satellites. Also, UE is equipped with an antenna array to receive $5 \mathrm{G}$ and GNSS signals.

The vector $\mathbf{p} \triangleq\left[p_{x}, p_{y}, p_{z}\right]^{\mathrm{T}}$ denotes the location of the $\mathrm{UE}$ in the 3-dimensional (3D) space, whereas the vector $\mathbf{q}_{i} \triangleq\left[q_{i x}, q_{i y}, q_{i z}\right]^{\mathrm{T}}$ refers to the $3 \mathrm{D}$ coordinate vector of a reference station, with $i=0$ for the $\mathrm{gNB}$ and $i>0$ for the satellites. We assume $\mathbf{q}_{i}$ is known, $\forall i$. Also, $\mathbf{q}_{0}$ is fixed, whereas $\mathbf{q}_{i}$ with $i>0$ changes with time, with velocity $\mathbf{v}_{i} \in \mathbb{R}^{3}$. Finally, we assume that the direction of the gNB's and UE's antenna boresight is indicated by the 3D orientation vectors $\mathbf{r}_{q}$ and $\mathbf{r}_{p}$, respectively. The direction $\mathbf{r}_{q}$ is known, whereas the relative orientation of $\mathbf{r}_{p}$ with respect to $\mathbf{r}_{q}$ can 
also be represented by the azimuth-elevation pair $(\alpha, \beta)$, which are unknown parameters to be determined.

The positioning of the UE is based on the joint processing of the received $5 \mathrm{G}$ and GNSS signals, respectively, denoted by $\mathbf{y}_{0}(t)$ and $\mathbf{y}_{s}(t)$. More specifically, $\mathbf{y}_{0}(t)$ is given by

$$
\begin{aligned}
\mathbf{y}_{0}(t)= & \sqrt{P_{g}} \mathbf{W}^{\mathrm{H}} \mathbf{H}\left(a_{0}, \theta_{0}, \psi_{0}, \phi_{0}, \xi_{0}, \alpha\right) \mathbf{F} \mathbf{x}_{0}\left(t-\tau_{0}\right) \\
& +\mathbf{W}^{\mathrm{H}} \mathbf{n}(t),
\end{aligned}
$$

where $P_{g}$ is the transmission power at the $\mathrm{gNB}, \mathbf{W} \in \mathbb{C}^{M \times M_{b}}$ and $\mathbf{F} \in \mathbb{C}^{N \times N_{b}}$ are the receive and transmit analog-digital hybrid beamforming matrices, $\mathbf{x}_{0}(t) \in \mathbb{C}^{N_{b}}$ is vector of $N_{b}$ reference signals transmitted by the $5 \mathrm{G}$ station, $\tau_{0}$ is the pathdelay defined as

$$
\tau_{0}=\varrho_{0}-\delta t
$$

where $\varrho_{0} \triangleq\left\|\mathbf{q}_{0}-\mathbf{p}\right\| / c$ is the time-of-flight, $c$ is the speedof-light, $\delta t$ is the UE's clock-bias with respect to the GNSS time $^{1}$ The $M \times N$ complex channel matrix is given by

$$
\mathbf{H}\left(a_{0}, \theta_{0}, \psi_{0}, \phi_{0}, \xi_{0}, \alpha\right)=a_{0} \mathbf{a}_{r, 0}\left(\theta_{0}, \psi_{0}, \alpha\right) \mathbf{a}_{t, 0}^{\mathrm{H}}\left(\phi_{0}, \xi_{0}\right),
$$

where $a_{0} \in \mathbb{C}$ denotes the channel coefficient, $\alpha$ is the rotation $^{2}, \theta_{0}, \psi_{0}$ and $\phi_{0}, \xi_{0}$ are the azimuth, elevation angles of the angle-of-arrival (AoA) and angle-of-departure (AoD), respectively. The vectors $\mathbf{a}_{r, 0}(\cdot) \in \mathbb{C}^{M}, \mathbf{a}_{t, 0}(\cdot) \in \mathbb{C}^{N}$ denote the receive and transmit steering vectors and are computed as,

$$
\begin{aligned}
\mathbf{a}_{r, 0}\left(\theta_{0}, \psi_{0}, \alpha\right) & \triangleq \exp \left(j \pi \mathbf{R}_{u}^{\mathrm{T}} \boldsymbol{\Gamma}^{\mathrm{T}}(\alpha) \boldsymbol{\pi}\left(\theta_{0}, \psi_{0}\right)\right), \\
\mathbf{a}_{t, 0}\left(\phi_{0}, \xi_{0}\right) & \triangleq \exp \left(j \pi \mathbf{R}_{g}^{\mathrm{T}} \boldsymbol{\pi}\left(\phi_{0}, \xi_{0}\right)\right),
\end{aligned}
$$

where $\boldsymbol{\pi}(a, b)$ is the unit-vector given by

$$
\boldsymbol{\pi}(a, b) \triangleq[\cos (b) \cos (a), \cos (b) \sin (a), \sin (b)]^{\mathrm{T}},
$$

$\mathbf{R}_{u} \in \mathbb{R}^{3 \times M}$ (similarly $\mathbf{R}_{g} \in \mathbb{R}^{3 \times N}$ ) is the antenna element location $^{3}$ matrix for the UE (and 5G-station), i.e.,

$$
\mathbf{R}_{u}=\left[\begin{array}{cccc}
0 & r_{1 x} & \cdots & r_{M x} \\
0 & r_{1 y} & \cdots & r_{M y} \\
0 & r_{1 z} & \cdots & r_{M z}
\end{array}\right],
$$

and, finally, the matrix $\Gamma(\alpha)$ is the rotation matrix given by

$$
\boldsymbol{\Gamma}(\alpha)=\left[\begin{array}{ccc}
\cos \alpha & -\sin \alpha & 0 \\
\sin \alpha & \cos \alpha & 0 \\
0 & 0 & 1
\end{array}\right]
$$

The received superposition of the GNSS signals is given by

$$
\mathbf{y}_{s}(t)=\mathbf{G}\left(\boldsymbol{\theta}_{s}, \boldsymbol{\psi}_{s}, \alpha\right) \mathbf{A}_{s} \mathbf{d}\left(t, \boldsymbol{\tau}_{s}, \mathbf{f}_{d}\right)+\mathbf{n}_{s}(t),
$$

where $\mathbf{y}_{s}(t) \in \mathbb{C}^{M_{s}}$ with $M_{s}$ as the number of GNSS antenna elements, $\mathbf{A}_{s} \in \mathbb{C}^{S \times S}$ is a diagonal matrix in which the $i i$-th element, $a_{i}$ is the complex amplitude of the received signal transmitted by the $i$-th satellite, $\boldsymbol{\theta}_{s} \triangleq\left[\theta_{1}, \cdots, \theta_{S}\right]$ and $\boldsymbol{\psi}_{s} \triangleq$ $\left[\psi_{1}, \cdots, \psi_{S}\right]$ are the azimuth and elevation vectors of the AoA

\footnotetext{
${ }^{1}$ The gNB is assumed perfectly synchronized with the GPS time.

${ }^{2}$ For simplicity, only a rotation around the $z$-axis is considered, i.e., $\beta=0$.

${ }^{3}$ Element locations are measured in wavelength unit and with respect to 1-st one (origin).
}

of the impinging satellite signals, the matrix $\mathbf{G}$ is referred to as spatial signature matrix and given by

$$
\mathbf{G}\left(\boldsymbol{\theta}_{s}, \boldsymbol{\psi}_{s}, \alpha\right) \triangleq\left[\mathbf{a}_{r, s}\left(\theta_{1}, \psi_{1}, \alpha\right), \cdots, \mathbf{a}_{r, s}\left(\theta_{S}, \psi_{S}, \alpha\right)\right],
$$

with

$$
\mathbf{a}_{r, s}\left(\theta_{i}, \psi_{i}, \alpha\right) \triangleq \exp \left(j \pi \mathbf{R}_{s}^{\mathrm{T}} \boldsymbol{\Gamma}^{\mathrm{T}}(\alpha) \boldsymbol{\pi}\left(\theta_{i}, \psi_{i}\right)\right),
$$

$\mathbf{R}_{s} \in \mathbb{R}^{3 \times M_{s}}$ denoting the GNSS antenna element location matrix and $\mathbf{d}\left(t, \boldsymbol{\tau}_{s}, \mathbf{f}_{d}\right) \in \mathbb{C}^{S}$ is the vector of delayed Dopplershifted GNSS signal with $d_{i}(t)$ given by

$$
d_{i}(t) \triangleq d_{i}\left(t, \tau_{i}, f_{d_{i}}\right)=x_{i}\left(t-\tau_{i}\right) e^{j 2 \pi f_{d_{i}} t},
$$

where each $x_{i}(t)$ is orthogonal to each other, and $\tau_{i}$ is

$$
\tau_{i}=\varrho_{i}-\delta t,
$$

with $\varrho_{i} \triangleq\left\|\mathbf{q}_{i}-\mathbf{p}\right\| / c$ and $f_{d_{i}}$ being the time-of-flight and Doppler shift between the UE and the $i$-th satellite.

\section{POSITION-ORIENTATION LOWER BOUND ANALYSIS}

In order to derive the position-orientation error for the hybrid 5G-GNSS positioning system, we compute the 5GGNSS EFIM as

$$
\mathbf{J}^{e}=\mathbf{J}_{0}^{e}+\mathbf{J}_{s}^{e},
$$

with $\mathbf{J}_{0}^{e}$ and $\mathbf{J}_{s}^{e}$ referring to the EFIM for the location parameters $\boldsymbol{\eta} \triangleq\left[\mathbf{p}^{\mathrm{T}}, \alpha, \delta_{t}\right]^{\mathrm{T}}$ obtained with the $5 \mathrm{G}$ and satellite signals, respectively.

The GNSS EFIM $\mathbf{J}_{s}^{e}$ is given by

$$
\mathbf{J}_{s}^{e}=\sum_{i=1}^{S}\left(\mathbf{J}_{s_{i}, \eta}-\mathbf{J}_{s, \eta a_{i}} \mathbf{J}_{s, a_{i}}^{-1} \mathbf{J}_{s, \eta a_{i}}^{\mathrm{T}}\right),
$$

where

$$
\begin{aligned}
& \mathbf{J}_{s_{i}, \eta}=\frac{1}{N_{0}} \int_{0}^{T_{s}} \Re\left\{\nabla_{\boldsymbol{\eta}}^{\mathrm{H}} \boldsymbol{u}_{s_{i}}\left(t, \boldsymbol{\eta}, a_{i}\right) \nabla_{\boldsymbol{\eta}} \boldsymbol{u}_{s_{i}}\left(t, \boldsymbol{\eta}, a_{i}\right)\right\} \mathrm{d} t, \\
& \mathbf{J}_{s_{i}, a_{i}}=\frac{1}{N_{0}} \int_{0}^{T_{s}} \Re\left\{\nabla_{a_{i}}^{\mathrm{H}} \boldsymbol{u}_{s_{i}}\left(t, \boldsymbol{\eta}, a_{i}\right) \nabla_{a_{i}} \boldsymbol{u}_{s_{i}}\left(t, \boldsymbol{\eta}, a_{i}\right)\right\} \mathrm{d} t,(17) \\
& \mathbf{J}_{s_{i}, \eta a_{i}}=\frac{1}{N_{0}} \int_{0}^{T_{s}} \Re\left\{\nabla_{\boldsymbol{\eta}}^{\mathrm{H}} \boldsymbol{u}_{s_{i}}\left(t, \boldsymbol{\eta}, a_{i}\right) \nabla_{a_{i}} \boldsymbol{u}_{s_{i}}\left(t, \boldsymbol{\eta}, a_{i}\right)\right\} \mathrm{d} t,
\end{aligned}
$$

where $T_{s}$ is the observation time of the GNSS signal, $\boldsymbol{u}_{s_{i}}\left(t, \boldsymbol{\eta}, a_{i}\right)$ is defined as

$$
\boldsymbol{u}_{s_{i}}\left(t, \boldsymbol{\eta}, a_{i}\right) \triangleq a_{i} \tilde{\mathbf{g}}_{i}(\mathbf{p}, \alpha) x_{i}\left(t-\tilde{\tau}_{i}(\mathbf{p})\right) e^{j 2 \pi \tilde{f}_{i}(\mathbf{p}) t}
$$

where $\tilde{\mathbf{g}}_{i}(\mathbf{p}, \alpha), \tilde{\tau}_{i}(\mathbf{p})$ and $\tilde{f}_{i}(\mathbf{p})$ indicate a re-parameterization of the $i$-th column of (10), the $i$-th path-delay (both measured with 5G and GNSS signals) and the $i$-th Doppler-frequency as a function of the location and rotation ${ }^{4}$.

\footnotetext{
${ }^{4}$ The Doppler frequency is also function of the UE's velocity, which is
} considered known and null, as UE is static. 
Similarly, the 5G-based EFIM is given by

$$
\mathbf{J}_{0}^{e}=\mathbf{J}_{0, \eta}-\mathbf{J}_{0, \eta a_{0}} \mathbf{J}_{0, a_{0}}^{-1} \mathbf{J}_{0, \eta a_{0}}^{\mathrm{T}},
$$

with $\mathbf{J}_{0, \eta}=\sum_{m=1}^{M_{b}} \mathbf{J}_{0 m, \eta}, \mathbf{J}_{0, a_{0}}=\sum_{m=1}^{M_{b}} \mathbf{J}_{0 m, a_{0}}$ and $\mathbf{J}_{0, \eta a_{0}}=$ $\sum_{m=1}^{M_{b}} \mathbf{J}_{0 m, \eta a_{0}}$ and $\mathbf{J}_{0 m, \eta}, \mathbf{J}_{0 m, \eta}$ and $\mathbf{J}_{0 m, \eta a_{0}}$ are computed as in (16), (17) and (18) by replacing $T_{s}$ with $T_{g}$ and $\mathbf{u}_{s_{i}}\left(t, \boldsymbol{\eta}, a_{i}\right)$ with

$$
u_{0 m}\left(t, \boldsymbol{\eta}, a_{0}\right) \triangleq \sqrt{P_{g}} \mathbf{w}_{m}^{\mathrm{H}} \tilde{\mathbf{H}}\left(\mathbf{p}, \alpha, a_{0}\right) \mathbf{F} \mathbf{x}_{0}\left(t-\tilde{\tau}_{0}(\mathbf{p})\right) e^{j 2 \pi t}
$$

where the subscript $m$ refers to the $m$-th baseband chain and $\tilde{\mathbf{H}}\left(\mathbf{p}, \alpha, a_{0}\right)$ is a re-parameterization of the 5G-based channel matrix as a function of the location and rotation. The reparameterization is explicited as follows

$$
\begin{aligned}
\theta_{i} & =\operatorname{atan}\left(\frac{p_{y}-q_{i y}}{p_{x}-q_{i x}}\right)-\pi, \\
\phi_{0} & =\operatorname{atan}\left(\frac{p_{y}-q_{0 y}}{p_{x}-q_{0 x}}\right), \\
\psi_{i} & =\operatorname{atan}\left(\frac{-\left(p_{z}-q_{i z}\right)}{\sqrt{\left(p_{x}-q_{i x}\right)^{2}+\left(p_{y}-q_{i y}\right)^{2}}}\right), \\
\xi_{0} & =\operatorname{atan}\left(\frac{p_{z}-q_{0 z}}{\sqrt{\left(p_{x}-q_{0 x}\right)^{2}+\left(p_{y}-q_{0 y}\right)^{2}}}\right), \\
\tau_{i} & =\frac{\left\|\mathbf{p}-\mathbf{q}_{i}\right\|}{c}-\delta_{t}, \\
f_{d_{i}} & =-\mathbf{v}_{i}^{\mathrm{T}} \mathbf{u}_{i} \frac{f_{c}^{s}}{c}, i>0
\end{aligned}
$$

where $c$ is the speed-of-light, $\mathbf{v}_{i}$ is the velocity vector of the $i$-th satellite, $f_{c}^{s}$ is the carrier frequency used of the satellite navigation system and

$$
\mathbf{u}_{i} \triangleq\left[\begin{array}{l}
u_{0 x} \\
u_{0 y} \\
u_{0 z}
\end{array}\right]=-\frac{\mathbf{p}-\mathbf{q}_{i}}{\left\|\mathbf{p}-\mathbf{q}_{i}\right\|} .
$$

\section{A. $5 G$ positioning EFIM}

In order to derive the EFIM for $5 \mathrm{G}$ positioning, we consider an orthogonal frequency-division multiplexing (OFDM) waveform with $N_{\text {FFT }}$ subcarriers and $\Delta_{f}$ subcarrier spacing. In the frequency domain the expression of the received signal, at the $n$-th subcarrier and at $m$-th beamformer is given by

$$
u_{0 m, n}=\sqrt{\frac{P_{g}}{N_{\mathrm{FFT}}}} a_{0} g_{d, n} g_{r, m} \boldsymbol{g}_{t}^{\mathrm{H}} \mathbf{x}_{n}+\mathbf{w}_{m}^{\mathrm{H}} \mathbf{n}_{n},
$$

where $g_{d, n} \triangleq e^{-j 2 \pi n \Delta_{f}}, g_{r, m} \triangleq \mathbf{w}_{m}^{\mathrm{H}} \mathbf{a}_{r}\left(\tilde{\theta}_{0}, \tilde{\psi}_{0}\right), \boldsymbol{g}_{t} \triangleq$ $\mathbf{F}^{\mathrm{H}} \mathbf{a}_{t}\left(\phi_{0}, \xi_{0}\right)$ and $\tilde{\theta}_{0}, \tilde{\phi}_{0}$ refers to the observed angle-of-arrival at the UE, which can be obtained from the identity

$$
\boldsymbol{\pi}\left(\tilde{\theta}_{0}, \tilde{\psi}_{0}\right)=\boldsymbol{\Gamma}(\alpha)^{\mathrm{T}} \boldsymbol{\pi}\left(\theta_{0}, \psi_{0}\right)=\boldsymbol{\pi}\left(\theta_{0}-\alpha, \psi_{0}\right) .
$$

The terms in (20) can be obtained from the block-matrix partion of

$$
\mathbf{J}_{0 m}=\mathbf{T}^{\mathrm{T}}\left(\sum_{n=1}^{N_{\mathrm{FFT}}} \tilde{\mathbf{J}}_{0 m, n}\right) \mathbf{T}
$$

where $\tilde{\mathbf{J}}_{0 m, n}$ is the FIM for the physical-channel parameters $\tilde{\boldsymbol{\eta}}_{g} \triangleq\left[\tau_{0}, \tilde{\theta}_{0}, \tilde{\psi}_{0}, \phi_{0}, \xi_{0}, \Re\left(a_{0}\right), \Im\left(a_{0}\right)\right]^{\mathrm{T}}$ and $\mathbf{T}$ is given by

$$
[\mathbf{T}]_{i j}=\frac{\partial \tilde{\eta}_{g, i}}{\partial \eta_{j}}
$$

in which the non-zero elements are given by

$$
\begin{aligned}
& \frac{\partial \tilde{\theta}_{0}}{\partial \mathbf{p}}=\frac{d}{d_{x y}^{2}}\left[u_{0 y},-u_{0 x}, 0\right], \\
& \frac{\partial \psi_{0}}{\partial \mathbf{p}}=\frac{1}{d_{x y}}\left[u_{0 x} u_{0 z}, u_{0 y} u_{0 z},-d_{x y}^{2} / d^{2}\right], \\
& \frac{\partial \tilde{\phi}_{0}}{\partial \mathbf{p}}=\frac{d}{d_{x y}^{2}}\left[u_{0 y},-u_{0 x}, 0\right], \\
& \frac{\partial \xi_{0}}{\partial \mathbf{p}}=-\frac{1}{d_{x y}}\left[u_{0 x} u_{0 z}, u_{0 y} u_{0 z},-d_{x y}^{2} / d^{2}\right], \\
& \frac{\partial \tau_{0}}{\partial \mathbf{p}}=-\frac{\mathbf{u}_{0}^{\mathrm{T}}}{c},
\end{aligned}
$$

and $\frac{\partial \tau_{0}}{\partial \delta_{t}}=1, \frac{\partial \tilde{\theta}_{0}}{\partial \alpha}=-1, \frac{\partial \Im\left\{a_{0}\right\}}{\partial \Im\left\{a_{0}\right\}}=1, \frac{\partial \Re\left\{a_{0}\right\}}{\partial \Re\left\{a_{0}\right\}}=1, d=\| \mathbf{p}-$ $\mathbf{q}_{0} \|, d_{x y}=\sqrt{\left(p_{x}-q_{0 x}\right)^{2}+\left(p_{y}-q_{0 y}\right)^{2}}$.

Finally, the FIM $\mathbf{J}_{0 m, n} \in \mathbb{R}^{8 \times 8}$ can be obtained by computing

$$
\mathbf{J}_{0 m, n}=\gamma_{g} \mathbb{E}_{x_{n}}\left\{\Re\left(\nabla_{\tilde{\boldsymbol{\eta}}_{g}}^{\mathrm{H}} u_{n m} \nabla_{\tilde{\boldsymbol{\eta}}_{g}} u_{n m}\right)\right\},
$$

where $\gamma_{g}=\frac{P_{g}}{N_{0} N_{N_{\mathrm{FFT}}} \Delta_{f}}, u_{n m}=a_{0} g_{d, n} g_{r, m} \boldsymbol{g}_{t}^{\mathrm{H}} \mathbf{x}_{n}$.

\section{B. GNSS positioning EFIM}

We focus on a Global Positioning System (GPS) technology with a carrier frequency $f_{c}^{s}$ with $N_{c}=1023$ chips of duration $T_{c}=1 / N_{c} \mathrm{~ms}$, i.e.,

$$
x_{i}(t)=\sqrt{P_{s_{i}}} \sum_{n=1}^{N_{c}} b_{i n} \operatorname{rect}_{T_{c}}\left(t-n T_{c}\right) e^{j 2 \pi f_{c}^{s} t},
$$

where $\operatorname{rect}_{T_{c}}(t)$ is the rectangular pulse of unitary amplitude and duration $T_{c}, P_{s_{i}}$ is the average transmission power and $\mathbf{b}_{i} \triangleq\left[b_{i 1}, \cdots, b_{i N_{c}}\right]$ is the code sequence transmitted by the $i$-th satellite. It is assumed $\mathbf{b}_{i}^{\mathrm{T}} \mathbf{b}_{q}=0, \forall i \neq q$.

Using equation (39) in (9) and by leveraging the orthogonality of the code sequences into the calculation of the EFIM, we compute $\mathbf{J}_{s}^{e}$ as in (15) where the matrices $\mathbf{J}_{s_{i}, \eta}, \mathbf{J}_{s, a_{i}}$ and $\mathbf{J}_{s, \eta a_{i}}^{\mathrm{T}}$ are obtained from the block-partition of

$$
\mathbf{J}_{s_{i}}=\mathbf{T}_{s_{i}}^{\mathrm{T}} \tilde{\mathbf{J}}_{s_{i}} \mathbf{T}_{s_{i}},
$$

with $\quad \tilde{\mathbf{J}}_{s_{i}}=\mathbb{E}\left\{\Re\left(\nabla_{\tilde{\boldsymbol{\eta}}_{s_{i}}}^{\mathrm{H}} u_{s_{i}} \nabla_{\tilde{\boldsymbol{\eta}}_{s_{i}}} u_{s_{i}}\right)\right\}, \quad \tilde{\boldsymbol{\eta}}_{s_{i}} \triangleq$ $\left[\tilde{\theta}_{i}, \tilde{\psi}_{i}, \tau_{i}, f_{d i}, \Re\left(a_{i}\right), \Im\left(a_{i}\right)\right]^{\mathrm{T}}$ and $\mathbf{T}_{s_{i}}$

$$
\left[\mathbf{T}_{s_{i}}\right]_{q j}=\frac{\partial \tilde{\eta}_{s_{i}, q}}{\partial \eta_{j}} .
$$




\section{Simulation RESUlts}

The objective of this section is to evaluate the performance of an hybrid 5G-GNSS positioning system and compare it to $5 \mathrm{G}$ and GNSS stand-alone solutions. In this regard, we consider a $5 \mathrm{G}-\mathrm{mmW}$ at $30 \mathrm{GHz}$, subcarrier spacing $60 \mathrm{kHz}$, transmission bandwidth $60 \mathrm{MHz}$, QPSK modulation and a GPS-based satellite positioning. Further details are in the sequel. UE: location coordinate $[0,0,0]^{\mathrm{T}} \mathrm{m}$, static, 5G uniform rectangular array (URA) $4 \times 4$ in the $z y$-plane, GPS receiver with URA $2 \times 2$ in the $x y$-plane, Discrete Fourier Transform (DFT) analog beam-codebook and sequential single beam-scanning. gNB: location coordinate $[-20,20,25]^{\mathrm{T}} \mathrm{m}$, 5G URA $8 \times 8$ in the $z y$-plane, DFT analog codebook and sequential single beam-scanning. Finally, GPS: observation time $T_{s}=20 \mathrm{~ms}$, bandwidth $B=4 / T_{c}$, carrier frequency $1575.42 \mathrm{MHz}$ and radius, azimuth and elevation directions of the satellites' locations

$$
\begin{aligned}
\boldsymbol{\varrho}_{s} & =[2.19,2.34,2.47,2.18,2.45] \times 1 \mathrm{e} 5 \mathrm{~km}, \\
\boldsymbol{\theta}_{s} & =[288.9,215.2,87.9,295.4,123.5] \mathrm{deg}, \\
\boldsymbol{\psi}_{s} & =[46.9,24.5,29.1,32.1,71.5] \mathrm{deg}
\end{aligned}
$$

The first result is shown in Figure 2 where the $x$ and $y$ axis refer to the achieved localization error by GNSS and 5G standalone. More specifically, for $5 \mathrm{G}$, we assume that $\mathrm{UE}$ is perfectly synchronized with gNB and the error is obtained by varying the $\mathrm{SNR}_{0}$ (i.e. the signal-to-noise ratio of the $5 \mathrm{G}$ signal without beamforming gain). For the GNSS, we assume that UE's clock-bias is non-zero and the performance depends on the carrier-to-noise $C / N_{0}$ (signal power to noise spectral density, typically used in GNSS). The contour plot corresponds to the hybrid 5G-GNSS solution obtained for all pairs $\left(\mathrm{SNR}_{0}, C / N_{0}\right)$ used for calculating $5 \mathrm{G}$ and GNSS bounds. It can be noticed that the hybrid method provides always a lower error than that obtained by a $5 \mathrm{G}$ or GNSS systems. Therefore, if one of the systems fails in terms of accuracy, the performance of an hybrid system takes advantage of the best between the two.

Next, we evaluate a more realistic scenario where UE clock is not synchronized with either 5G or GNSS, and the clock bias is unknown. Figures 3 and 4 show the localization error achieved with 5 and 3 satellites, respectively. The black-solid line corresponds to the performance of the GNSS, whereas the colored dash and solid lines indicate $5 \mathrm{G}$ system with perfectly synchronized UE's clock and hybrid 5G-GNSS system with unknown UE's clock bias, respectively.

In both simulations, it can be noticed that the proposed 5GGNSS method is always more accurate than the GPS 5 but, only after a certain carrier-to-noise value, it is better than a perfectly synchronized 5G system. In other words, this study indicates that exists a certain value of the carrier-to-noise signal, beyond which a practical $5 \mathrm{G}$ positioning can benefit of the interaction

\footnotetext{
${ }^{5}$ GPS positioning with three satellites is theoretically feasible by using an antenna array and observing the Doppler shifts at the receiver since the dependence of the AoA and Doppler with the position is exploited. However, errors are very large for realistic $C / N_{0}$.
}

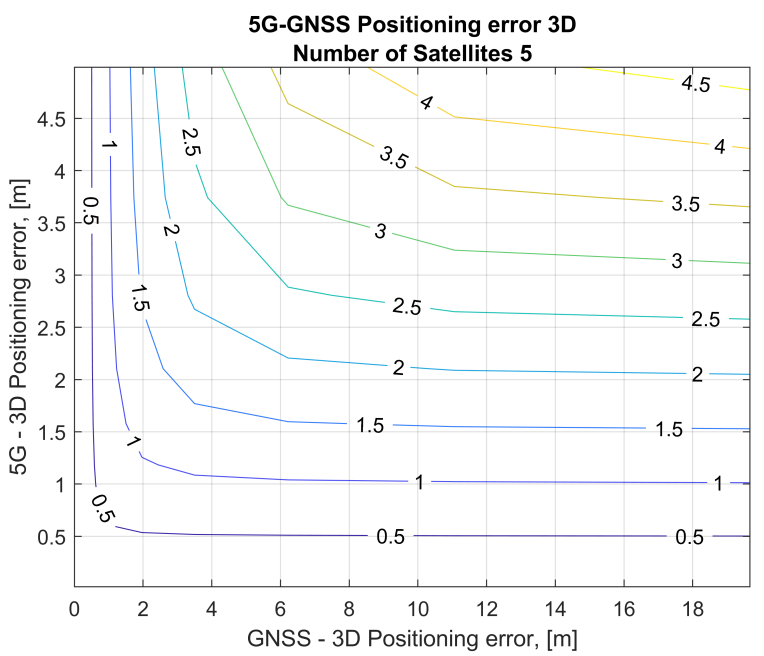

Fig. 2. Contour plot of the hybrid system with perfectly synchronized UE.

with GNSS to improve accuracy. However, this value varies with the number of satellites by comparing Figures 3 and 4 .

Figure 5 illustrates the estimation error of the UE clock-bias. It can be noticed that no performance is shown for the standalone $5 \mathrm{G}$ system as the UE clock-bias is not identifiable with mere LOS measurements. However, both GNSS and hybrid 5G-GNSS can provide an estimate of the bias that improves with carrier-to-noise. The benefits of a joint processing of 5G and GNSS observables are clearly remarked by the lower error achieved by this solution, which is more accentuated in the low carrier-to-noise regime and less at unrealisticly high carrier-to-noise ratios. Comparing this result with the location error shown in Figure 4, a tight correlation can be identified which, for instance, can be exploited for a mutual performance improvement by using side information of location or synchronization.

Next, in Figure 6 the estimation error of the UE's rotation is provided. In this case, the performance is plotted as a function of the 5G Signal-to-Noise Ratio (SNR) as we are interested to a direct comparison of to the orientation error with $5 \mathrm{G}$. Again, the advantage of the hybrid positioning is proved as the achievable error is lower than the best of the two solutions.

In contrast to the location error, the 5G-GNSS performance lines do not intersect the others. This indicates that there is no minimum carrier-to-noise requirement, since the hybrid solution always improves the orientation estimation.

Finally, as an example, let us mention that a specific positioning requirement, e.g. $50 \mathrm{~cm}$, can be achieved by either with a very good GNSS configuration ( 5 satellites and $C / N_{0}=57 \mathrm{dBHz}$ ) or by a hybrid solution with at least 3 satellites with $C / N_{0}=60 \mathrm{dBHz}, 5 \mathrm{G}$ SNR $-14 \mathrm{~dB}$. By increasing the 5G SNR, weaker requirements on GNSS signals can be imposed.

\section{CONCLUSION}

In this paper we studied the fundamental limits of 5GGNSS solution for positioning, orientation and UE's clock- 


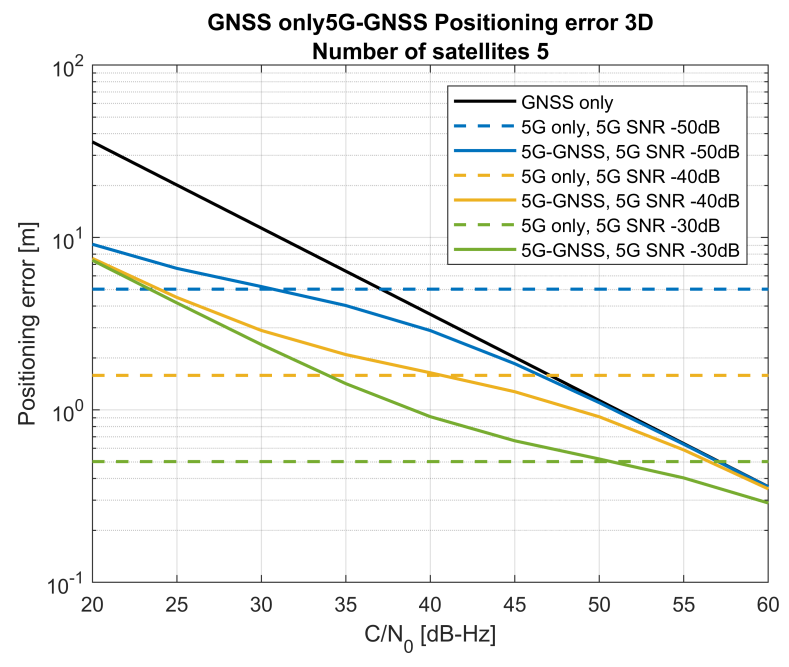

Fig. 3. Behaviour of a 5G-GNSS position error as a function of the GNSS carrier-to-noise and using the GNSS observables from 5 satellites.

GNSS only5G-GNSS Positioning error 3D

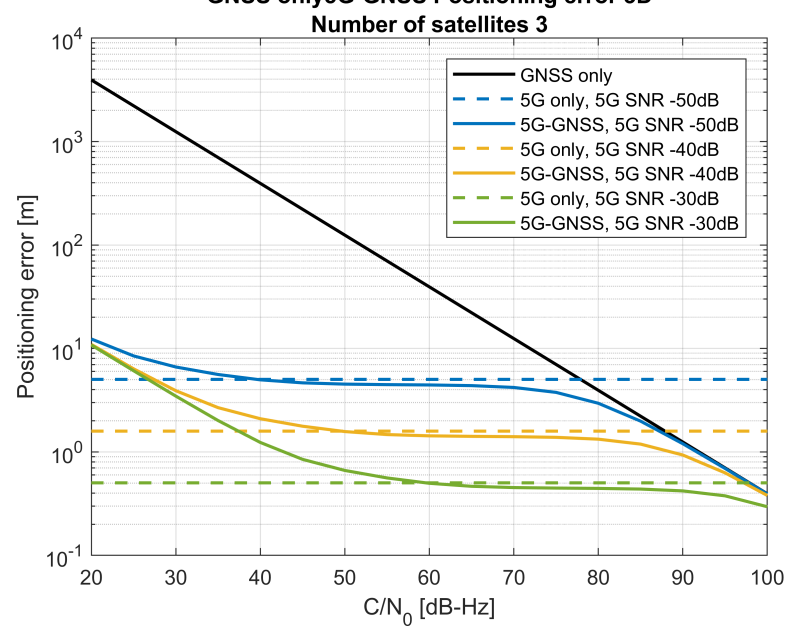

Fig. 4. Behaviour of a 5G-GNSS position error as a function of the GNSS carrier-to-noise and using the GNSS observables from 3 satellites.

bias estimation. It was found that, with a tight coupling of the observables, GNSS benefits from $5 \mathrm{G}$ to improve localization accuracy and reduce the number of connected satellites. On the other hand, $5 \mathrm{G}$ takes advantage of a GNSS system to improve gNB-UE synchronization issues as well as to achieve better localization and orientation accuracy in the low SNR regime. The Doppler, observed only in GNSS measurements, was useful to improve the accuracy of the GNSS system.

\section{ACKNOWLEDGMENTS}

This work was supported, in part, by the Academy of Finland projects 6Genesis Flagship (grant 318927), Fundamental of Simultaneous Localization and Communications (grant 298781), and Positioning-aided Reliably-connected Industrial Systems with Mobile mmWave Access (PRISMA), by the EU H2020 project 5GCAR, and the VINNOVA COPPLAR project, funded under Strategic Vehicle Research and Innovation Grant No. 2015-04849 and R+D Project of Spanish Min-

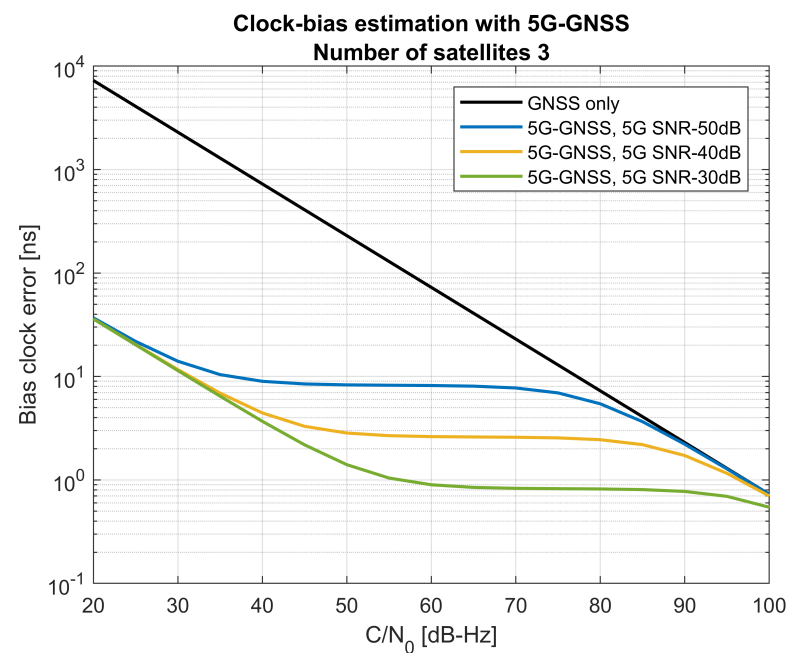

Fig. 5. Behaviour of a clock-bias error as a function of the GNSS carrier-tonoise and using the GNSS observables from 3 satellites.

5G-GNSS orientation error 3D

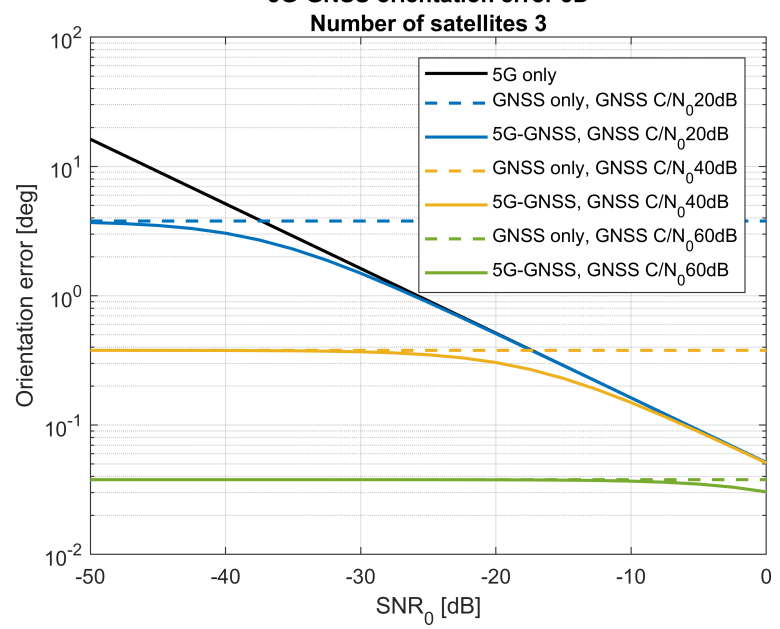

Fig. 6. Behaviour of a UE orientation error as a function of the $5 \mathrm{G}$ SNR (without beamforming) and using the GNSS observables from 3 satellites.

istry of Economy and Competitiveness under Grant TEC201789925-R.

\section{REFERENCES}

[1] H. Wymeersch, G. Seco-Granados, G. Destino, D. Dardari, and F. Tufvesson, "5G mmwave positioning for vehicular networks," IEEE Trans. Wireless Commun., vol. 24, no. 6, pp. 80-86, Dec. 2017.

[2] E. S. Lohan, M. Koivisto, O. Galinina, S. Andreev, A. Tolli, G. Destino, M. Costa, K. Leppanen, Y. Koucheryavy, and M. Valkama, "Benefits of positioning-aided communication technology in high-frequency industrial IoT," IEEE Commun. Mag., pp. 1-7, 2018.

[3] P. J. G. Teunissen and O. Montenbruck, Handbook of Global Navigation Satellite Systems. Springer, 2017.

[4] A. Shahmansoori, G. E. Garcia, G. Destino, G. Seco-Granados, and H. Wymeersch, "Position and orientation estimation through millimeterwave MIMO in 5G systems," IEEE Transactions on Wireless Communications, vol. 17, no. 3, pp. 1822-1835, Mar. 2018.

[5] J. del Peral-Rosado, J. Saloranta, G. Destino, J. Lòpez-Salcedo, and G. Seco-Granados, "Methodology for Simulating 5G and GNSS HighAccuracy Positioning," Sensor, vol. 18, no. 10, 2018.

[6] S. M. Kay, Fundamentals of Statistical Signal Processing: Estimation Theory. Upper Saddle River, NJ, USA: Prentice-Hall, Inc., 1993. 